\title{
Reinventing the Medical Assistant Staffing Model at No Cost in a Large Medical Group
}

\author{
Nadim M. Ilbawi, $M D^{1,2}$ \\ Monica Kamieniarz, RN MSN ${ }^{1}$ \\ Avisek Datta, MS ${ }^{1}$ \\ Bernard Ewigman, MD, MSPH ${ }^{1,2}$ \\ 'Department of Family Medicine, NorthShore University Health- \\ System, Lincolnwood, Illinois \\ ${ }^{2}$ University of Chicago, Chicago, Illinois \\ Ann Fam Med 2020;18:180. https://doi.org/10.1370/afm.2468.
}

\section{THE INNOVATION}

Mounting nonclinical burdens, declining staff ratios, and rotating staff in large medical groups increase burnout and hamper the joy of primary care practice. ${ }^{1}$ Some systems invest heavily in additional staff and training to offload this nonclinical work from physicians. ${ }^{2-5}$ We reorganized our existing medical assistant (MA) staffing model from rotating assignments to a matched pairing of 1 MA to each physician. We created a structured approach to personalize methods of improving efficiency, productivity, quality, and job satisfaction for each pair.

\section{WHO \& WHERE}

One primary care practice in NorthShore University Health System, a large US integrated health system.

\section{HOW}

We matched 7 MAs each with 1 of the 5 family physicians or 2 internists to work as a pair guided by preferred choices ranked by both the MAs and the physicians. Each pair completed a standardized workbook to personalize office workflows, task delegation, communication patterns, and schedule management.

The workbook was used as the initial framework to guide the continuity working relationships. Familiarity with the physician's clinical and communication preferences for typical work performed by the MAs such as repeat blood pressure measurements, strep tests, urine tests, immunizations, and EKGs led to

\footnotetext{
Conflicts of interest: authors report none.
}

\section{CORRESPONDING AUTHOR}

Nadim Ilbawi, MD

NorthShore University Health System

Department of Family Medicine

6810 N. McCormick Blvd.

Lincolnwood, IL 60712

nilbawi@northshore.org greater efficiencies. Each MA knew when to perform these tasks based on chief complaint, whether the physician was on schedule or not, and clinician preference (eg performing strep tests before vs after physician evaluation). This greatly reduced delays and decreased the communication needed for each patient. These efficiencies as well as preclinic huddles allowed options for double booking and other same day schedule changes which improved the workday flow substantially. Additionally, this created a more coordinated team-based experience for patients.

As trust increased due to better shared understanding, physicians also delegated more tasks that had not been routinely performed by these MAs in the rotating model. Examples include pending orders for appropriate screening tests and labs, assistance with physician in-basket management, and follow-up directly with patients to assure adherence to agreedupon care plans. Some physicians also entrusted their MAs to close the visit by summarizing after-visit care instructions.

Finally, all MAs assisted directly with achieving quality metrics. All MAs continued working full time (40 hours per week) and synchronized schedules with their physician. Full-time physicians had 30 to 34 scheduled patient hours; MAs therefore had 6 to 10 hours of nonpatient visit hours devoted to improving quality metrics and other patient outreach. Examples of tasks performed included scheduling follow-up appointments, documenting completed preventive-care measures (screening procedures and laboratory tests) that were completed outside our system, and following up on home blood pressure readings. In the rotating staff model, physicians performed most if not all of these additional clerical tasks.

\section{LEARNING}

By comparing the 6 months before this change to the 6 months following the intervention in this practice as well as using 29 similar primary care practices in our system as a control group, we found that our one-on-one continuity model offloaded some nonclinical work, provided greater physician control of the practice micro-environment, improved physician productivity (11\% average increase in relative value units (RVUs) per physician) and significantly improved hemoglobin $A_{1 c}$ values, cervical cancer screening rates, and depression screening rates. Physicians reported increased satisfaction and observed an enhanced sense of personal accountability and investment in success by the MA often lacking in the rotating staff model.

For additional information, including references, key words, funding support, and prior presentations, see it online at http:II www.AnnFamMed.org/content/18/2/180/suppl/DC1/. Workbook available from author upon request. 